\title{
Histology of Coronary Arteries in Relation to the Coronary Sclerosis
}

\section{Pranjali $\mathrm{R}$ and Sachin $\mathrm{S}^{*}$ \\ Assistant Professor, Department of Anatomy, Government Medical College, India Assistant Professor, Department of Anatomy, Government Medical College, India}

*Corresponding author: Dr. Sachin Shembekar, Assistant Professor, Department of

\section{Research Article}

Volume 2 Issue 2

Received Date: June 20, 2018

Published Date: June 29, 2018 Anatomy, Government Medical College, Plot no. 169/170, Flat no. 101, Shri Gajanan apartment, Shri Mahalakshmi Nagar, New Narsala road, Nagpur 440034, India, Tel: 8087253751; Email: sachinshembekar21@rediffmail.com

\section{Abstract}

Coronary arteries were dissected out from 112 human hearts including 62 males and 50 females in all age groups. Various parameters of coronary sclerosis were studied in three major coronary arteries namely right coronary artery (RCA), left anterior descending coronary artery (LAD) and left circumflex coronary artery in both the sexes (LCX). The histological sections were stained with H \& E stain whereas special stain (Aldehyde Fuschine) was used to stain internal elastic lamina. Thickness of artery wall and the diameter of coronary artery were measured with the help of ocular micrometer. Among the three major coronary arteries age related evidence of coronary sclerosis is more in Left anterior descending coronary artery followed by right coronary artery and left circumflex coronary artery with least affected. Whatever the criteria used for measurement, coronary sclerosis is more common in male as compared to female in all age groups.

Keywords: Coronary arteries; Coronary sclerosis; Aldehyde fuschine; Internal elastic lamina; Ocular micrometer

\section{Introduction}

Coronary sclerosis has been studied intensively since so many years, but is still poorly understood. The investigation of coronary artery sclerosis in clinical material is hampered by the inaccessibility of the coronary arteries. They cannot be directly examined during life. Clinical evidence of coronary disease generally occurs only with an advanced process, and then the symptoms may not be proportional to the degree of involvement of arteries.
ET Bell [1] has stated that the most prominent influence in the development of coronary sclerosis is age. The commonly accepted belief is that coronary disease develops in later life and that it progresses steadily with age [2]. Coronary sclerosis may affect young persons. White, Edwards, and Dry [3] found no linear progression in severity of coronary sclerosis in men past 50. Clawson and Bell [4] also found no increase in the percentage of those dying of coronary disease beyond the fifth decade. 
Clinically, only $1.7 \%$ of all coronary disease occurs before the age of 40 [5]. This fact has produced the popular conception that coronary sclerosis is a disease only of more advanced age. A more difficult aspect of age in relation to coronary disease is the question of the age at which the process starts. Several authors have reported changes occurring in the coronary arteries during the early months of life $[6,7]$. The question has been whether these observed changes are pathological or are merely developmental changes in the arterial wall. The changes appear to blend imperceptibly into recognizable atherosclerosis [8].

The study to be reported here is based upon the examination of post-mortem material. The primary objective has been determination of effect of age on the incidence and severity of coronary sclerosis and determination of the age at which the sex difference in incidence may begin first. General histological architecture of coronary artery is shown in Photograph-I.

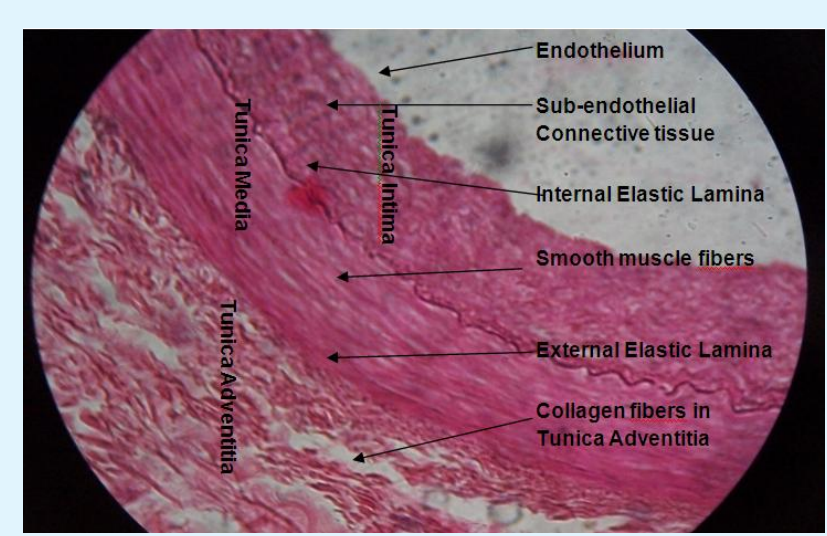

Figure 1: Showing Normal Histological Structure of Coronary Arterial Wall.

\section{Material and Methods}

The study consisted of thorough examination of coronary arteries which were dissected out from 112 human hearts including 62 males and 50 females (GraphI) removed at medico legal post-mortems done within 6 hours of death during a period of 3 years $(2013,2014$, and 2015). The study was carried out in the histology lab department of anatomy GMC Nagpur after taking prior permission from local ethical committee of government medical college, Nagpur. These Hearts were taken randomly specially from persons killed by accidental means from all age groups. The study material was divided in 8 age groups with a range of 10 years (GraphII).

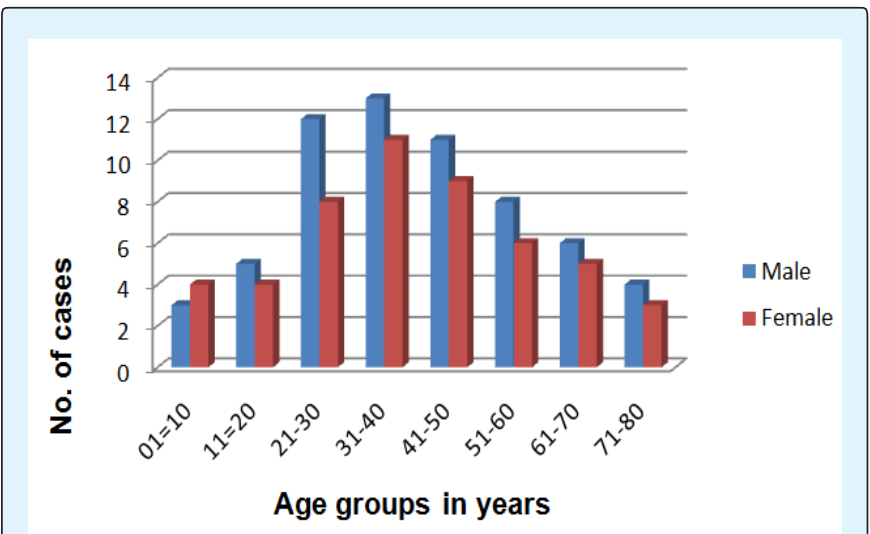

Graph 1: Bar diagram showing distribution of cases according to age and sex.

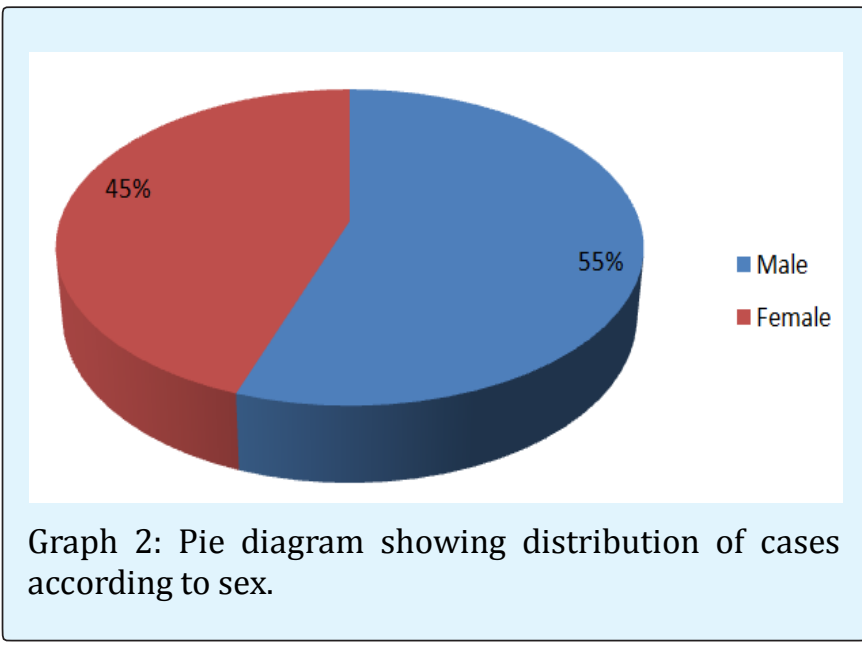

Tissue samples were obtained from right coronary artery (RCA) and left anterior descending coronary artery (LAD) and left circumflex coronary artery (LCX) $2 \mathrm{~mm}$ away from their origin. Multiple paraffin blocks were prepared and 7-8 micron thick sections were obtained. The slides were stained with H\&E stain. Aldehyde fuschine stain was used to stain internal elastic lamina [9].

Wall thickness and diameter of the coronary arteries were measured with the help of ocular micrometer (Photograph-II) circumferentially at 8 different places along the planes at $45^{\circ}$ to each other and then their mean was taken as a reading for the respective artery. 


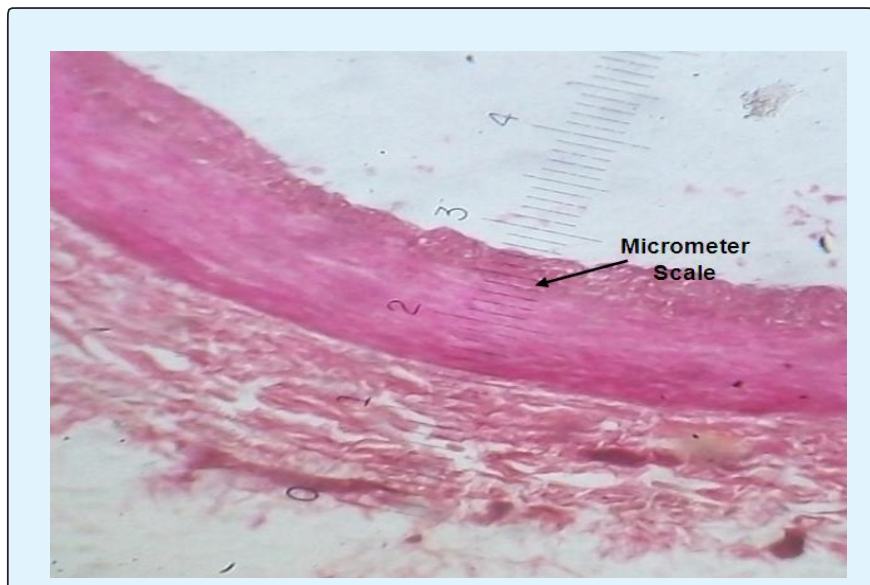

Figure 2: Showing Micrometer Scale.

The degree of degeneration of internal elastic lamina was graded independently from 0 to 4 . The numerical grades of 0 to 4 for each of the three major coronary arteries were added together so that numerical grade of 0 to 12 was obtained without having fraction. Grade-1 (Photograph-III) consisted of the first visible splitting of internal elastic lamina. Grade-2 (Photograph-IV) was applied to those arteries which showed actual breaks in the continuity of the lamina but in which the lamina could be followed around more than one half of the circumference of the lumen. Grade-3 (Photograph-V) was applied when less than one half of the lumen was still surrounded by lamina and Grade-4 (Photograph-VI) when no recognisable internal elastic lamina was found.

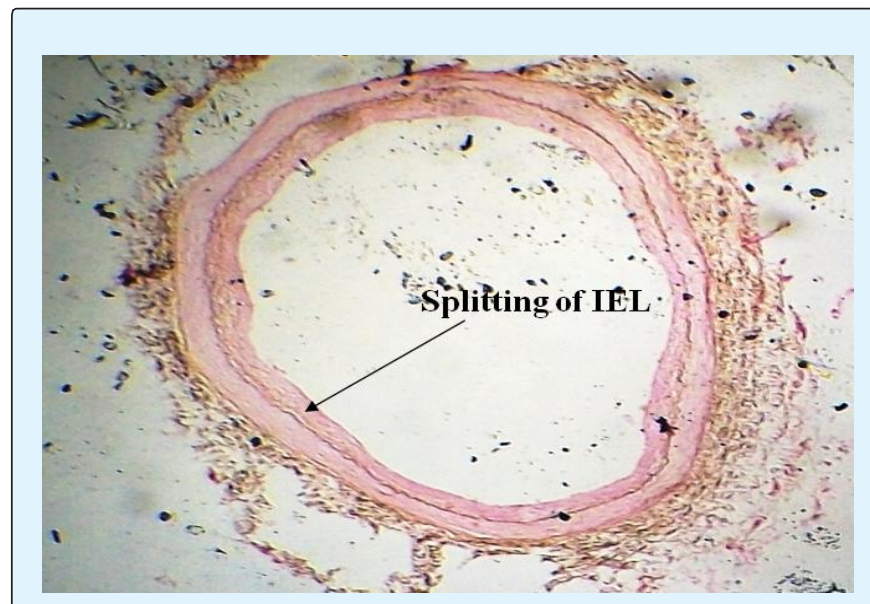

Figure 3: Showing Grade-I Degeneration of Internal Elastic Lamina (IEL).

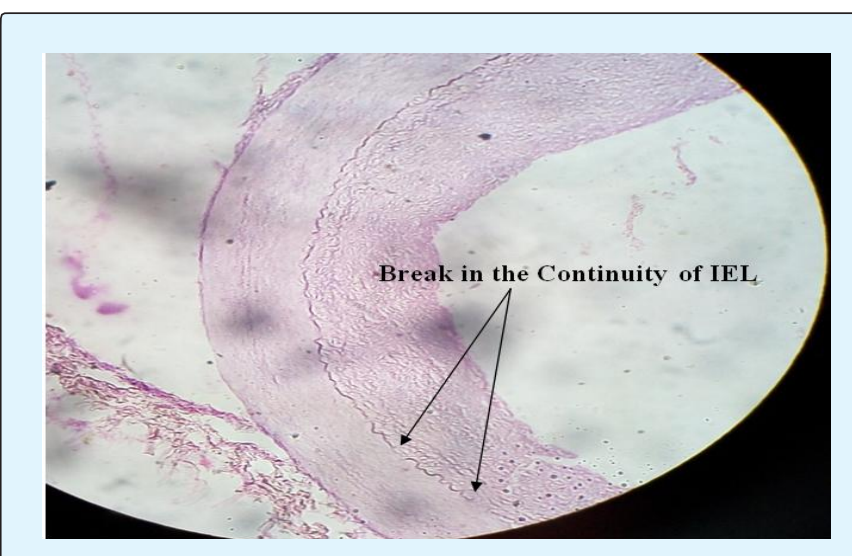

Figure 4: Showing Grade-II Degeneration of Internal Elastic Lamina (IEL).

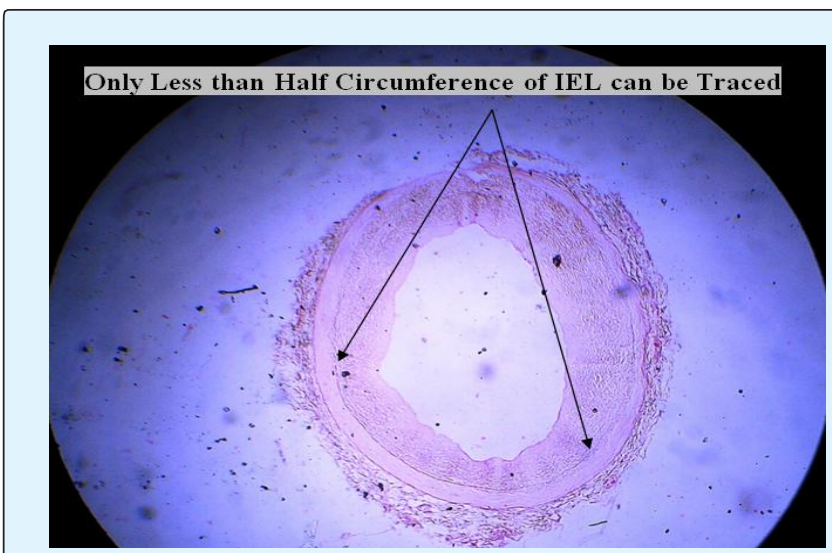

Figure 5: Showing Grade-III Degeneration of Internal Elastic Lamina (IEL).

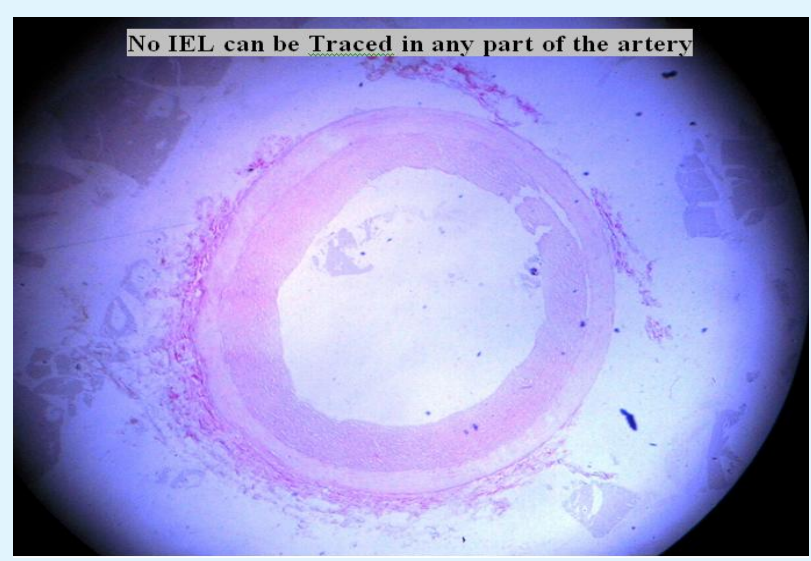

Figure 6: Showing Grade-III Degeneration of Internal Elastic Lamina (IEL). 


\section{Observations}

We observed that when average relative area of lumen as percentage of area of artery is compared then there is a progressive decrease in the area of lumen in coronary arteries (Graph-III).
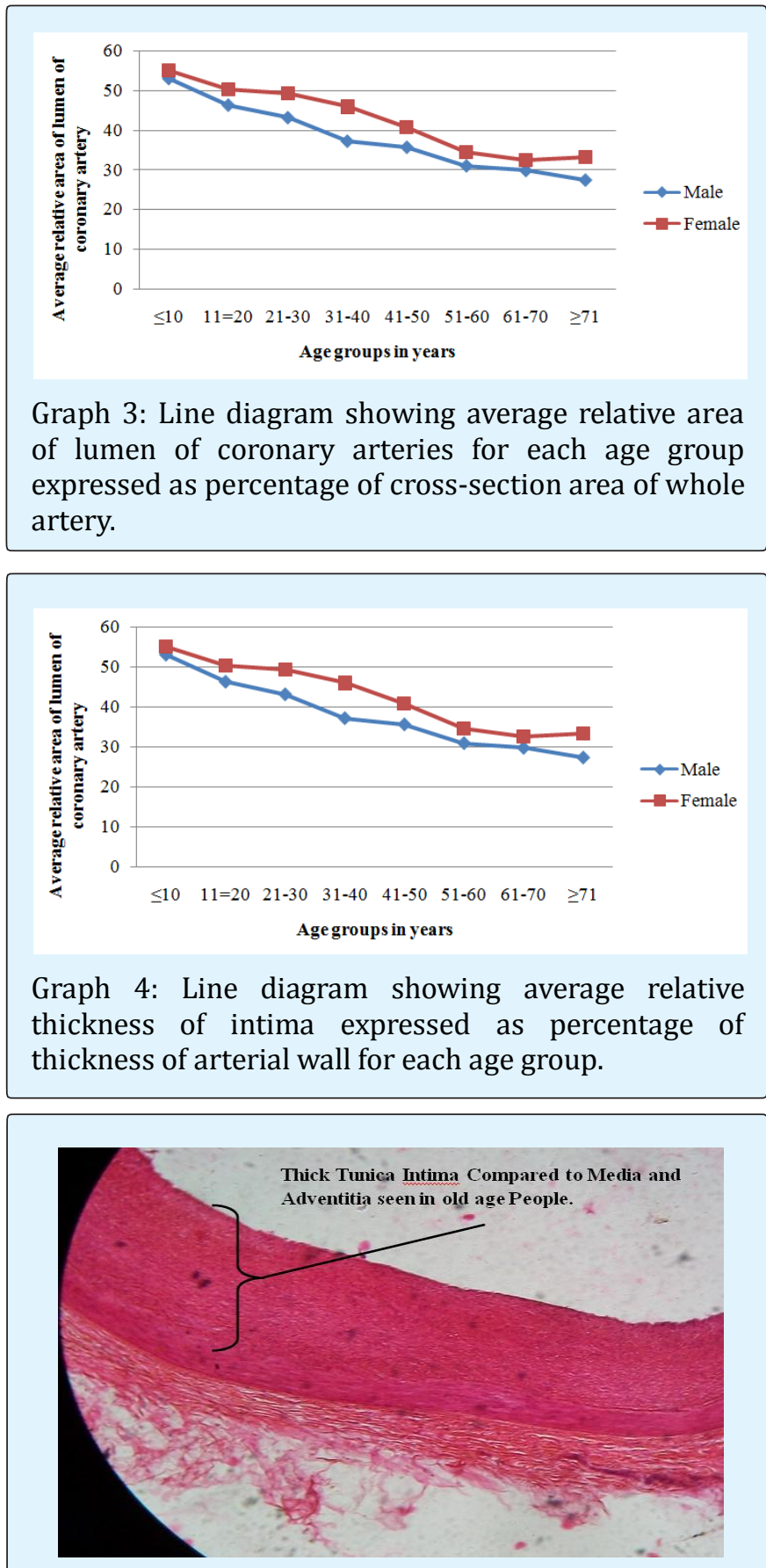

Figure 7: Showing Coronary Artery of Male in Eighth Decade.

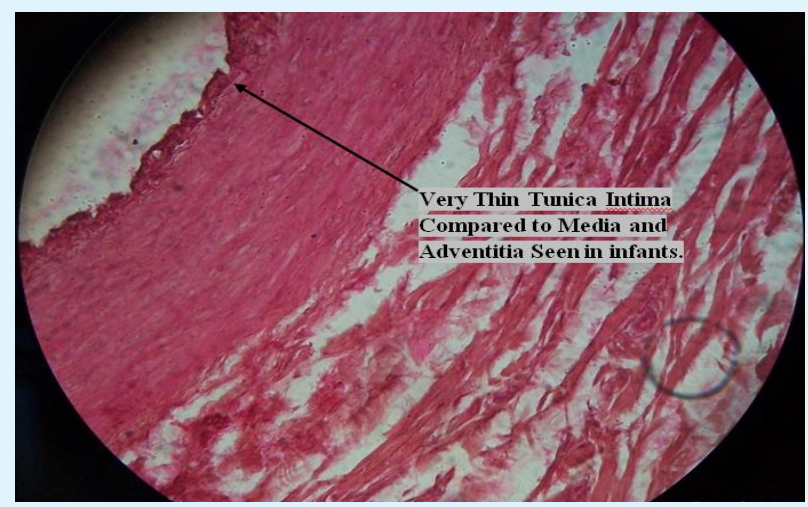

Figure 8: Showing Coronary Artery of Female in First Decade.
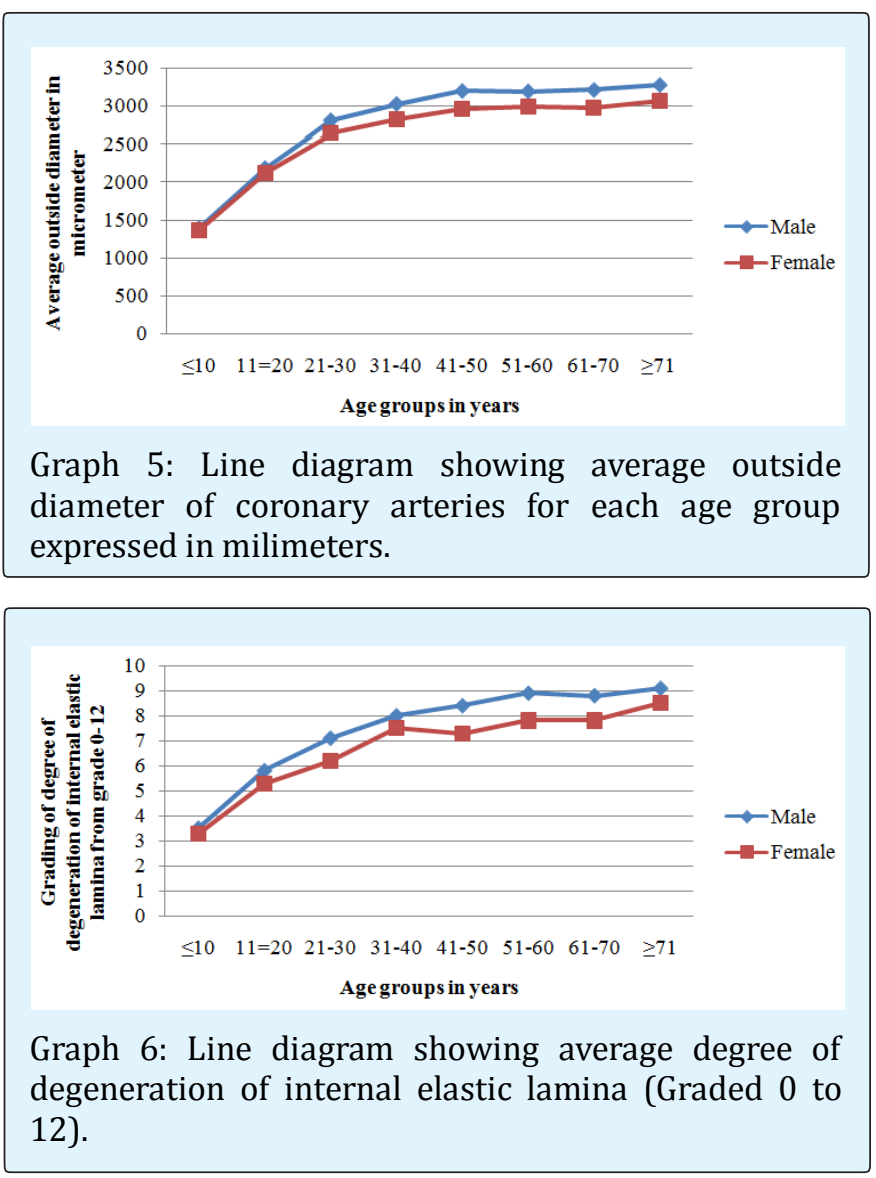

It was also observed that when average thickness of intima as percentage of thickness of artery wall was compared, and plotted on a graph, there was progressive increase in intimal thickness in the coronary arteries (Graph-IV), (Photograph-VII \& VIII). 
When outside diameter was plotted in a similar manner it was unexpected to find the size of the artery increasing throughout life (Graph-V).

The average degree of internal elastic lamina degeneration for each heart showed figure ranging from 3.3 in first decade girl to 9.1 in men in the ninth decade. The curve plotted from these figures showed continuously increasing slope (Graph-VI).

Regarding sex deference whatever the criteria used for measurement, the male were seen to have considerably more changes of atherosclerosis in every decade than female (Graph III-VI).

\section{Discussion}

Results obtained from this study indicate that coronary sclerosis appears to be a continuous process which is inseparable from the 'developmental' changes observed in children. The terminal decline in the severity is probably due to the survival of resistant individuals into the eighth decade. The number of hearts from persons which were examined in this decade was small and so definite conclusion cannot be drawn. The terminal decline has also been noted by White, Edwards, and Dry [3].

The results of this study suggest that there is progressive increase in thickness of tunica intima of coronary arteries and also there is continuous increase in the degree of degeneration of internal elastic lamina. Similar findings were observed by other investigators [10-12]. It is the intimal thickening that eventually leads to the coronary sclerosis and carries the seeds of modern epidemic of coronary artery diseases.

The results of the present study suggest that whatever the parameters used to calculate, age is the most important factor in coronary sclerosis, a finding which confirms the idea of previous investigators. A gradual progression of changes from childhood to old age is contrary to the generally accepted belief that coronary artery disease begins in middle age or later.

It is somewhat unexpected that diameter of coronary arteries continue to increase throughout life observed in the present study. A similar increase was noted by Ehrich, de la Chapelle, and Cohn [7] in the coronary arteries and other vessels. The enlargement probably represents dilatation or loss of elasticity rather than continued growth, at least in older age groups.
Severity of coronary sclerosis is different between males and females. This difference is shown to begin in early childhood, and continue till old age groups $[12,13]$. It is of sufficient magnitude to place females from 10-20 years "behind" males in the development of coronary sclerosis. Once the process is established, however, the rate of progression of coronary sclerosis appears to be nearly same in both the sexes. The beginning of sex difference before the age of puberty suggests that hormonal differences may not be the important factors. This concept is also suggested by the maintenance of the difference beyond the menopausal age group.

The work of Dock [14] and Fangman and Hellwig [8] the arteries of infants has suggested the beginning of measurable differences between male and female coronary arteries before birth. This suggestion has yet to be proved. Whether the early thickening of the intima and splitting of the internal elastic lamina are considered developmental or pathological, they are continuous with the process of adult life, as are the other changes in the artery.

The work of Hamilton [15] has shown that the male of nearly all species studied, including man, has a shorter life span and is more susceptible to more injurious processes than the female, from the time of conception onward. It seems probable that coronary sclerosis should also come under this heading, and that the underlying cause of sex difference lies in this inborn basic difference between the sexes and not in any of the more superficial factors which can be studied. He attributes the mechanism of the difference to a generally more rapid metabolism in male.

The severity of the lesions in the three main branches of the coronary arteries of each heart was generally found to be of about the same grade. The process in most cases was slightly advanced in the left main and anterior descending branch, with the right coronary artery being second, and the left circumflex coronary artery least commonly involved. However, the differences were slight. This finding is similar to that of most other investigators $[3,4,7]$.

\section{Conclusion}

The coronary sclerosis appears to be a continuous process that is inseparable from developmental changes that is observed early in infants and children. The slowly progressive nature of the process throws some doubt upon theories which attempt to relate the fundamental 
cause of atherosclerosis to conditions occurring first in adult life.

The difference in severity of coronary sclerosis between males and females is shown to begin in early childhood and continue till old age.

The comparison of various methods of grading the severity of the coronary sclerosis demonstrated that the gradually progressive character of the process was shown by any of the methods used. The intima thickens, the lumen is reduced, the artery dilates and the internal elastic lamina degenerates at about the same rate. This observation does not necessarily hold true for the individual case, as there is considerable individual variation.

When death or symptoms from coronary disease had occurred the heart showed severe sclerosis of all the three main arteries, no matter at what age the person died. There was an average reduction of the relative area of the lumen to less than $30 \%$ of that of the artery. This observation indicates that people must have severe coronary disease before it causes clinical symptoms. Thus there appears to be a large reserve capacity in the coronary system.

Conflict of interest: None

Source of funding: Self

Ethical clearance: Done by local ethical committee of government medical college Nagpur.

Acknowledgement: Nil

\section{References:}

1. Bell ET (1934) Relation of Arteriosclerosis to Senescence. Am J Path 10: 705.

2. Piersol GM (1946) Coronary Artery Disease in the Aging. Clinics 4: 1114-1123.

3. White NK, Edwards JE, Dry TJ (1950) The Relationship of the Degree of Coronary Atherosclerosis with Age in Men. Circulation 1: 645654.

4. Clawson BJ, Bell ET (1949) Incidence of Fatal Coronary Disease in Non-Diabetic and in Diabetic Persons. Arch Path (Chic) 48(2): 105.

5. Glendy RE, Levine SA, White PD (1937) Coronary
Disease in Youth: Comparison of 100 Patients Under 40 with 300 Persons Past 80. JAMA 109(22): 17751781.

6. Bork K (1926) Uber Kranzadersklerose. Arch Path Anat 262(3): 646-657.

7. Ehrich W, de la Chapelle C, Cohn AE (1931) Anatomical Ontogeny B. Man; I. A Study of the Coronary Arteries, Am J Anat banner 49(2): 241-282.

8. Fangman RJ, Hellwig CA (1947) Histology of Coronary Arteries in Newborn Infants. Am J Path 23(5): 901.

9. Singh DR (2003) Principles and technique in histology, microscopy and photomicrography. $1^{\text {st }}$ edition. New Delhi and Bangalore. CBS publishers and distributers 152-156.

10. Crawford T (1976) Symmers systeric pathology chapter I and II A $2^{\text {nd }}$ (Edn.), volume I. London. Churchill livingstone 8: 120-123.

11. Tyagi SP, Dadgar SK (1978) Histological changes in coronary arteries in relation to age. Indian Heart J 30(4): 246-248.

12. Sachin S, Meena M (2016) Age Related Histological Changes in Tunica Intima of Coronary Arteries in Relevance to the Atherosclerosis IJFMT JulyDecember 10(2): 168-171.

13. Dhall U, Chaudhary S, Sirohiwal BL (2003) Histomorphometric analysis of coronary arteries sexual dimorphism. J Anat society of India 52(2): 144146.

14. Dock W (1946) The Predilection of Atherosclerosis for the Coronary Arteries. JAMA 131(11): 875-878.

15. Hamilton JB (1948) The role of testicular secretions as indicated by the effect of castration in man and by studies of pathological conditions and the short lifespan associated with maleness. Recent Prog Hormone Research 3: 257-322.

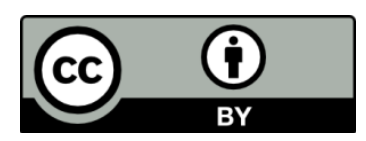

